\title{
REACHABILITY OF NASH EQUILIBRIA IN COMPETITIVE LOCATION MODELS
}

\author{
H.A. Eiselt, University of New Brunswick, Canada \\ J. Bhadury, University of New Brunswick, Canada
}

In this paper we present a competitive location model. Models of this type have been used since Hotelling (1929) in his seminal paper "Stability in Competition" suggested a scenario in which duopolists compete on a linear market, i.e. a line segment, in prices and locations. His conclusion was that an equilibrium exists in which both competitors locate at the center of the market. This result was dubbed the "principle of minimum differentiation" and has been used by various authors to explain why existing products and political platforms are so similar to each other.

It was not until 1979, fifty years after the original paper was published, that d'Aspremont et al. discovered that the Hotelling model did, in fact, not possess an equilibrium at all. The underlying reason is that while the possibility of capturing additional markets between the two facilities forces them together, intense price competition à la Bertrand forces them apart. The authors also showed the volatility of the model: replacing the original linear transportation costs by quadratic ones results in an equilibrium in which competitors locate as far apart as possible, i.e. maximum, rather than minimum, differentiation.

What followed was a plethora of different models, most of them slight variations on the original theme. Even if only the major contributions are counted, Eiselt et al. (1993) have classified more than hundred of them. Some of the models possess equilibria while others don't, emphasizing the extreme sensitivity of the model. The approach in this paper focuses on a different aspect of equilibria rather than their existence. In short, we consider a model in which duopolists have fixed prices and compete only in locations. The original locations of the two competitors are arbitrary. Each competitor will now relocate considering his opponent's location as fixed. In this locational game we distinguish between two cases, a game with sequential moves and one in which competitors relocate simultaneously. Assuming that a locational equilibrium exists, our question is whether or not the equilibrium will always be reached in such a relocation game.

In the model under consideration, the market is in the shape of a tree with customers, whose demand for the homogeneous good is fixed, located at the vertices. The two competitors charged fixed, but not necessarily equal, mill prices, so that customers are responsible for the transport cost. Customers buy from the competitor with the lowest delivered price, i.e. mill price plus transport cost.

The case of equal prices is easy. Locational equilibria always exist and are always reached. If prices are unequal, it is shown that existing equilibria may actually be missed. However, we demonstrate that with an appropriate tie-breaking rule, existing equilibria will always be found. 